\title{
A BICYCLE ROBOT: PART 2 SYSTEM IMPLEMENTATION
}

\author{
P. Sooraksa, T. Uthairat, S. Kaopratum, U. Sritheerawirojana, \\ and V. Monyakul*
}

\author{
Department of Industrial Technology and Information Engineering, \\ Faculty of Engineering, \\ King Mongkut's Institute of Technology Ladkrabang, \\ Chalongkrung Rd., Ladkrabang,Bangkok, 10520,Thailand \\ Email: kspitikh@kmitl.ac.th \\ *National Science and Technology Development Agency, \\ Ministry of Science Technology and Environment, \\ 73/1 Rama Vi Rd., Rajdhevee, Bangkok, 10400, Thailand \\ Email:veerapol@mozart.inet.co.th
}

\begin{abstract}
This paper presents the realization and implementation of a bicycle robot using the control scheme designed in Part 1. The control hardware architecture of the robot consists of tilt sensors, a turning control unit, a driving unit and a microcontroller. In a control cycle, the functional mechanism can be described as follows: First, the tilt sensors sense the state of the robot's balance position and then send the signal to the microcontroller for generating a control decision based on the control law. After the final fuzzy control decision has been made, the output signal will be sent to the turning and the driving control units to track the desired path while retaining the robot in balance.
\end{abstract}

Keywords: bicycle robot, fuzzy logic control, modeling, intelligent control, two wheeled robot

\section{INTRODUCTION}

Implementation of a bicycle robot is a challenge topic in robotics and control engineering. This type of robot can be considered as an inherently unstable, nonlinear, and complex plant. This paper takes that challenge to be granted. Moreover, it is more challenge to build the successful system with a limit budget, "off-the-shelf" motors and other used hardware components. In doing so, a bicycle robot named "Robobike" is designed by two of the authors in [1], and is then built the real system as presented in Figure 1. Two small supporting wheels shown in all Figures appeared in this paper are used for safety reason and will be taken away during the test.

Since a bicycle has an inverted pendulum-like feature in the sense that it will fall into the ground if no control action from the rider or absence of a supporting stand, one might think that this problem can be solved easily by using a technique appeared in a control textbook. Unfortunately, the real world is neither always linearized nor predictable in all cases, even the simulation shows a satisfactory result.

For instance, one may realize a built-in gyro-servo regulator available in the market places as a sensor and an actuator to take care of the balance task for the system. Such an implementation seems to be finished because the gyroscope as a tilt sensor can detect the roll angle of the robot and the servo inside it can send the control signal to control the robot. Moreover, the gyro-servo popularly used for controlling a hobby vehicle such as a small helicopter has a balance regulator inside. With a DC motor to drive a rear wheel, the hardware components would be perfectly fit the control system as suggested by the simulation.

Like most of the cases, the simulation often performs under a certain assumption for sake of simplicity and convenience. The above hardware realization as suggested by simulation cannot accomplish the overall system performance because of lacking of consideration for robustness. For example, in the bad road condition, a little stone on the road can make the robot falling down to the road. 
The abrupt disturbance as an unforeseen input disturbance could go beyond the threshold or capability of such a built-in gyro-servo regulator.

In addition, the above realization is not able to retain a balance at the first moment of the operation because the torque of the built-in gyro-servo for small helicopter is too small to drive a handlebar of the bicycle. Hence, the torque needs to be amplified by adding a bigger servomotor to produce adequate torque. As we have seen some limitation of using the simplified simulation alone for hardware implementation. Nevertheless, the simulation result informs us about the problem in hand is feasible to implement, but more robustness and the practical consideration need to be enhanced. The issue is presented in the next section.

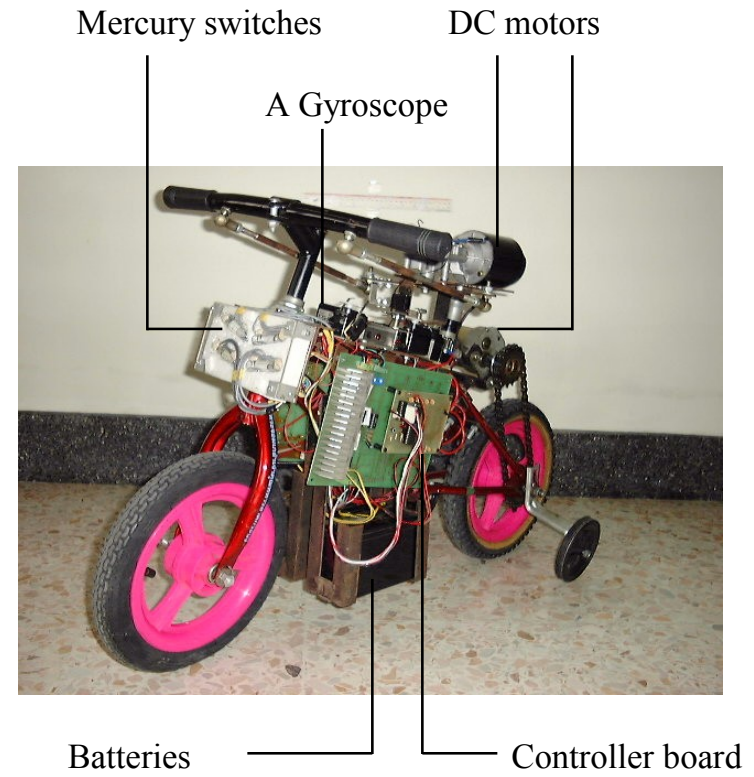

Figure 1. Robobike: A bicycle robot.

\section{OVERALL SYSTEM STRUCTURE}

\subsection{Control Algorithm}

"Robobike" is designed to operate in two modes, which are automatic and manual modes. In the manual mode, the robot is controlled remotely by radio signals. In the automatic mode, the control specifications are that the robot can track a target route while retaining balance. The control architecture is depicted in Figure 2.

The control hardware architecture of the robot consists of tilt sensors, a turning control unit, a driving unit and a microcontroller. The system operates in accordance with the selecting mode. In the automatic mode, a control cycle can be described as follows: First, the tilt sensors sense the state of the robot's balance position and then send the signal to the microcontroller for generating a control decision based on the control law. After the final fuzzy control decision has been made, the output signal will be sent to the turning and the driving control units to track the desired path while retaining the robot in balance.

The tilt sensors are a gyroscope and mercury switches. We use these sensors for roll angle measurement. The gyroscope used here is SRM-206 SG-X of SANWA equipped with a servomotor SRM1323. We add mercury switches to cope with the situation that the gyroscope cannot detect the monotonically small rate of change of the lean angle. We installed four mercury switches in $8^{\circ}, 13^{\circ}, 172^{\circ}$, and $167^{\circ}$ orientation with respect to the horizontal line in the counterclockwise sense. For more smooth operation, one can add more mercury switches as desired. In the case of a tire of the robot hitting on a small stone and the gyro-servo mechanism cannot deal with the balance, one of the switches will be activated to inform the microcontroller. The control action based on the fuzzy control law will be urgently performed.

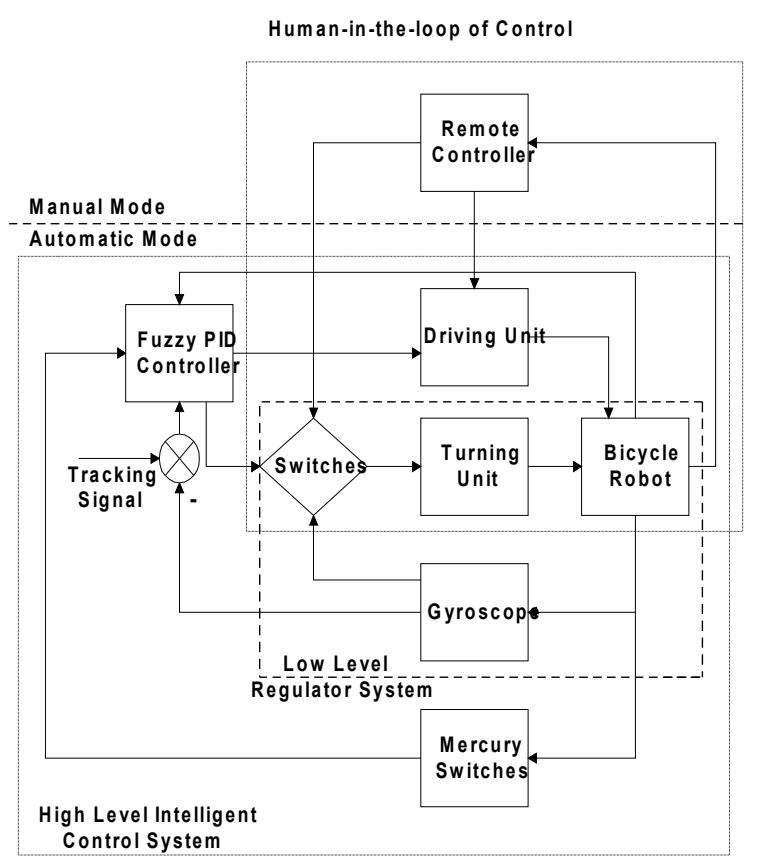

Figure 2. Control architecture.

According to Figure 2., the fuzzy PID controller for tracking a given path and retaining a target and a standard engineering approach called hierarchical control structure are realized. The fuzzy PID control is implemented in the form of software in Assembly language. To reduce the microcontroller cost, we use AT89C52 produced by Atmel [2]. The microcontroller operates at the frequency of 11.0592 $\mathrm{MHz}$. The microcontroller controls a turning unit by 
receiving 4 bits of a signal from the mercury switches via Port 1 and then send the control signal through the same port using the rest of the bits.

\subsection{Power Supply}

Energy source of the robot comes from two 12Volt-DC batteries connected in series produced the total $24 \mathrm{~V}$. Since there are many types of electronic circuits used in to control the robot, the switching supply circuit is required to transform the $24 \mathrm{~V}$ into required voltages corresponding to various circuits. Block diagram for switching power supply is shown in Figure 3. From Figure 3, the buck converter is not only used to transform the $24 \mathrm{~V}$ into $13 \mathrm{~V}$ for the turning unit, but also used for supplying power of $5 \mathrm{~V}$ 1 A via IC L4960 to the microcontroller and mercury switches. To get the buck converter started up, we used IC UC3842 to control the current mode for power MOSFET of the converter.

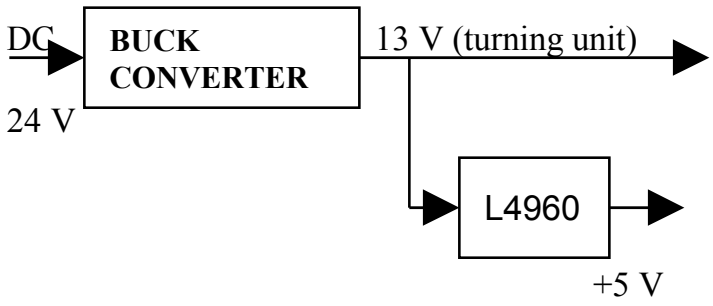

Figure 3. Switching power supply

\section{MAIN COMPONENTS}

\subsection{Driving Unit}

The main device of this unit is a $78 \mathrm{rpm} 18 \mathrm{~V} \mathrm{DC}$ motor with 1:30 gear block. We use IC L200 of SGS-Thomson as a programmable voltage regulator. The input of the control circuit is the voltage of $24 \mathrm{~V}$ from the batteries and the output is transformed into $18 \mathrm{~V}$ for the motor. A relay of $5 \mathrm{~A} 12 \mathrm{~V}$ is used to make the state of ON and OFF for the voltage output to drive the motor.

\subsection{Turning Unit}

Two wire-wound potentiometers are used as sensors for identifying position of the servomotor - one for the input of the turning circuit and the other for the feedback position. Figure 4 shows the installation of the potentiometers.

In this subsystem, a linear power amplifier type is used as a servo amplifier for improving and amplifying the control signal before feeding to the 12 V 3A DC servomotor of TOYOTA TG 159100953A. The driver of the motor is a Motorola IC MC33030 [3]. Since the IC specification at the
Power H-switch requires only $1 \mathrm{~A}$ while the servo needs 3 A. Hence the output of MC33030 at Port 14 and 15 need to be modified by connecting a power Hswitch buffer (we used transistors TIP 2955 and TIP 3055 of SGS Thompson).

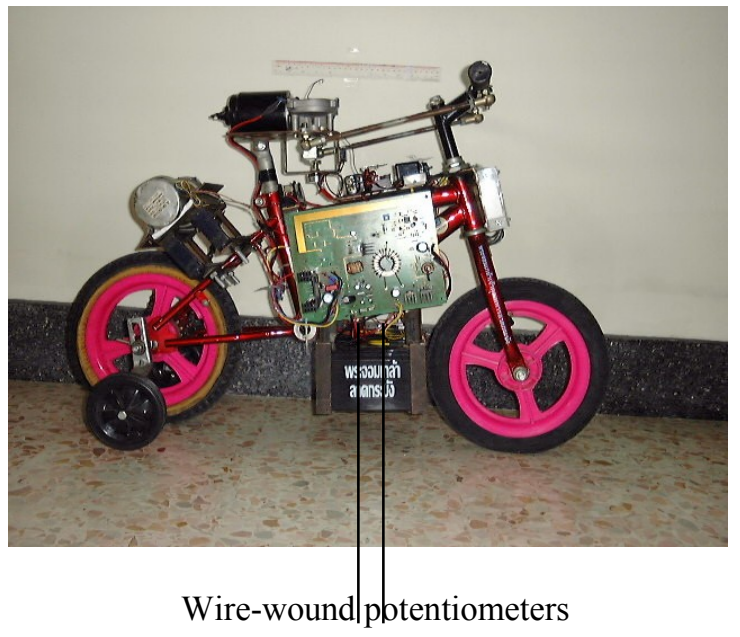

Figure 4. The side view of Robobike presents wire-wound potentiometers.

From the aforementioned about the above turning unit, the top view of the subsystem is shown in Figure 5.

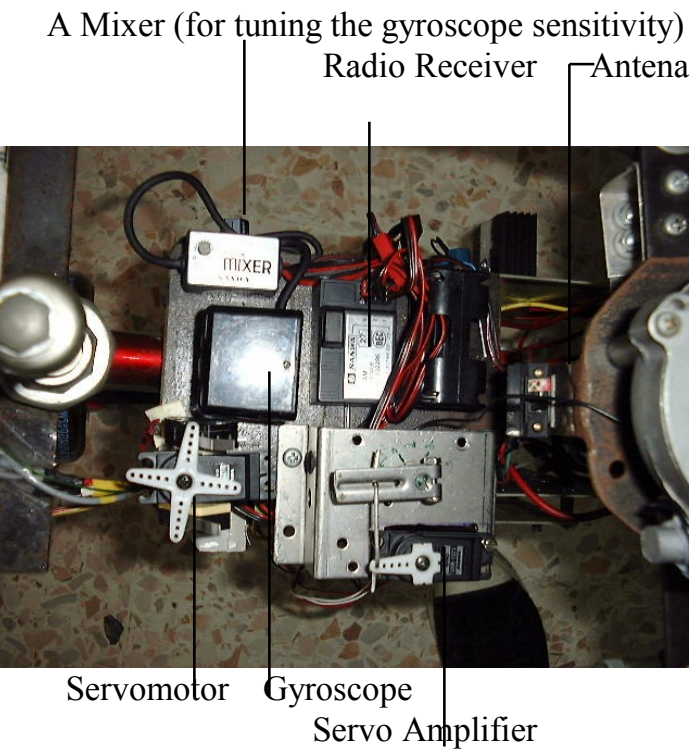

Figure 5. The top view of the turning unit.

\section{CURRENT LIMITATION AND FUTURE WORK}

Based on our testing, the Human-in-the-loop of Control yields a satisfactory result after we gain experience on controlling the robot via radio control. In this manual mode, like any other radio control 
system, the more time and afford we play with the system, the more expertise we become.

Although the manual mode is successfully built, the automatic mode is still on trouble-shooting processes. With the intelligent fuzzy logic based features, the pulse encoding for control fuzzification is under working to seek for the appropriate membership function and its components quantitatively. Once the suitable membership parameters and the control parameters are clearly identified physically and experimentally, the fuzzy PID architecture will be implemented in the form of a computer program. In short, the implementation of the hardware is almost finished, whereas the software is still on going development.

\section{CONCLUSION}

We have presented the implementation of a bicycle robot in this paper. As the first attempt to control this type of robot, the manual mode is first built and the satisfactory result is obtained. The next step along with the challenge is still in the light of getting the automatic mode works well as in the simulation. Integration of collision avoidance sensors such as ultrasonic and infrared sensors is in the consideration for the future work.

\section{Acknowledgments}

The first author is financially supported by the Thailand Research Fund.

\section{REFERENCES}

[1] Sooraksa, P., and Sritheerawirojana, U., "A Bicycle Robot: Part 1 Modeling and Control," $17^{\text {th }}$ IAARC/IFAC/IEEE Intl. Symp. on Automation and Robotics in Construction, 2000.

[2] Atmel, AT89C52 8-Bit Microcontroller with $8 \mathrm{~K}$ Bytes Flash, 1997.

[3] Motolora, MC33030 DC Servo Motor Controller /Driver, Motolora, Inc., 1996. 